\title{
Syncope Caused by Nonsteroidal Anti-Inflammatory Drugs and Angiotensin-Converting Enzyme Inhibitors
}

\author{
Chinori Kurata, MD; Akihiko Uehara, MD; \\ Toshihiko Sugi, MD; Keisuke Yamazaki, MD
}

\begin{abstract}
A 85-year-old woman with diabetes mellitus and prior myocardial infarction was transferred to the emergency room with loss of consciousness due to marked bradycardia caused by hyperkalemia. The $\mathrm{T}$ wave during right ventricular pacing was tall and tent-shaped while the concentration of serum potassium was high, and its amplitude during pacing was decreased after correction of the serum potassium level. Simultaneously with the correction, normal sinus rhythm was restored. The cause of hyperkalemia was considered to be several doses of loxoprofen, a nonsteroidal anti-inflammatory drug (NSAID), prescribed for her lumbago by an orthopedic specialist, in addition to the long-term intake of imidapril, an angiotensin-converting enzyme inhibitor (ACEI), prescribed for her hypertension by a cardiologist. This case warns physicians that the combination of NSAID and ACEI can produce serious side effects in aged patients who frequently suffer from hypertension, diabetes mellitus, ischemic heart disease, and degenerative joint disease. (Jpn Circ J 1999; 63: 1002-1003)
\end{abstract}

Key Words: Angiotensin-converting enzyme inhibitors (ACEI); Elderly; Hyperkalemia; Nonsteroidal antiinflammatory drugs (NSAID); Syncope

$\mathbf{M}$ arked bradycardia can be caused by hyperkalemia, and both nonsteroidal anti-inflammatory drugs (NSAID) and angiotensin converting enzyme inhibitors (ACEI) may cause hyperkalemia. To our knowledge, however, there has been no case report in which syncope due to marked bradycardia may have been caused by a combination of a NSAID and an ACEI. In addition, it has not been described whether the so-called 'tent-shaped' $T$ wave due to hyperkalemia may be observed during right ventricular pacing.

We report a case in which syncope was caused by the combination of a NSAID and an ACEI, and in which the peaked $\mathrm{T}$ wave was induced by hyperkalemia during right ventricular pacing as well as during spontaneous rhythm.

\section{Case Report}

A 85-year-old woman was transferred to the hospital emergency room with loss of consciousness due to marked bradycardia (Fig 1). She had hypertension, non-insulindependent diabetes mellitus and hypercholesterolemia, which were all controlled with the use of several drugs for several months before admission. Her blood pressure was 130-150/60-80 mmHg, HbA1c 5.2-6.0\%, and serum total cholesterol $5.17-5.95 \mathrm{mmol} / \mathrm{L}(200-230 \mathrm{mg} / \mathrm{dl})$. She also had a history of acute anterior myocardial infarction at the age of 81 years.

On the day of admission, she took breakfast as usual. From $11.00 \mathrm{~h}$ she began to get ready to go out, while complaining of mild lightheadedness. At about $11.50 \mathrm{~h}$ she felt dizzy and lay in bed. At noon she became drowsy,

(Received June 23, 1999; revised manuscript received August 23, 1999; accepted September 7, 1999)

Department of Medicine III, Hamamatsu University School of Medicine, Hamamatsu, Japan

Mailing address: Chinori Kurata, MD, Department of Medicine III, Hamamatsu University School of Medicine, 3600 Handa-cho, Hamamatsu 431-3192, Japan. E-mail: kurata-c@ post.yamaha.co.jp vomited, and then made no response to the calls of her family. At $12.30 \mathrm{~h}$ she arrived at hospital by ambulance. She showed no reaction when her name was called, but responded to painful stimuli, and showed marked bradycardia with a downward tendency in blood pressure. Temporary right ventricular pacing was immediately started and she promptly recovered consciousness. A laboratory examination of peripheral venous blood revealed no elevation of serum cardiac muscle enzymes, but there was hyperkalemia $(7.4 \mathrm{mmol} / \mathrm{L})$, which was considered to cause the marked bradycardia. Glucose and insulin were intravenously administered to correct the hyperkalemia and it decreased to $4.1 \mathrm{mmol} / \mathrm{L}$ within $3 \mathrm{~h}$. Simultaneously with the reduction in the serum potassium level, normal sinus rhythm at a rate of about 70 beats/min was restored and the amplitude of $\mathrm{T}$ waves was decreased. The $\mathrm{T}$ wave during right ventricular pacing was tall and tent-shaped while the concentration of serum potassium was high. Its amplitude during right ventricular pacing also decreased after the normalization of serum potassium level (Fig 2).

The cause of hyperkalemia was considered to be several doses of loxoprofen, a NSAID, prescribed for her lumbago by an orthopedic specialist, in addition to her long-term intake of imidapril, an ACEI, prescribed for her hypertension by a cardiologist. Indeed, the concentrations of serum potassium and creatinine increased from $4.6 \mathrm{mmol} / \mathrm{L}$ and $66.3 \mu \mathrm{mol} / \mathrm{L}(0.75 \mathrm{mg} / \mathrm{dl})$, respectively, immediately before the start of NSAID administration to $7.4 \mathrm{mmol} / \mathrm{L}$ and $99.9 \mu \mathrm{mol} / \mathrm{L}(1.13 \mathrm{mg} / \mathrm{dl})$ on admission. Cessation of both drugs was sufficient to maintain the normal level of serum potassium and normal sinus rhythm during and after her convalescence.

\section{Discussion}

Hyperkalemia may cause various electrocardiographic changes; for instance, the tent-shaped $\mathrm{T}$ wave, depression of the ST segment, decrease in the amplitude of the $\mathrm{R}$ 


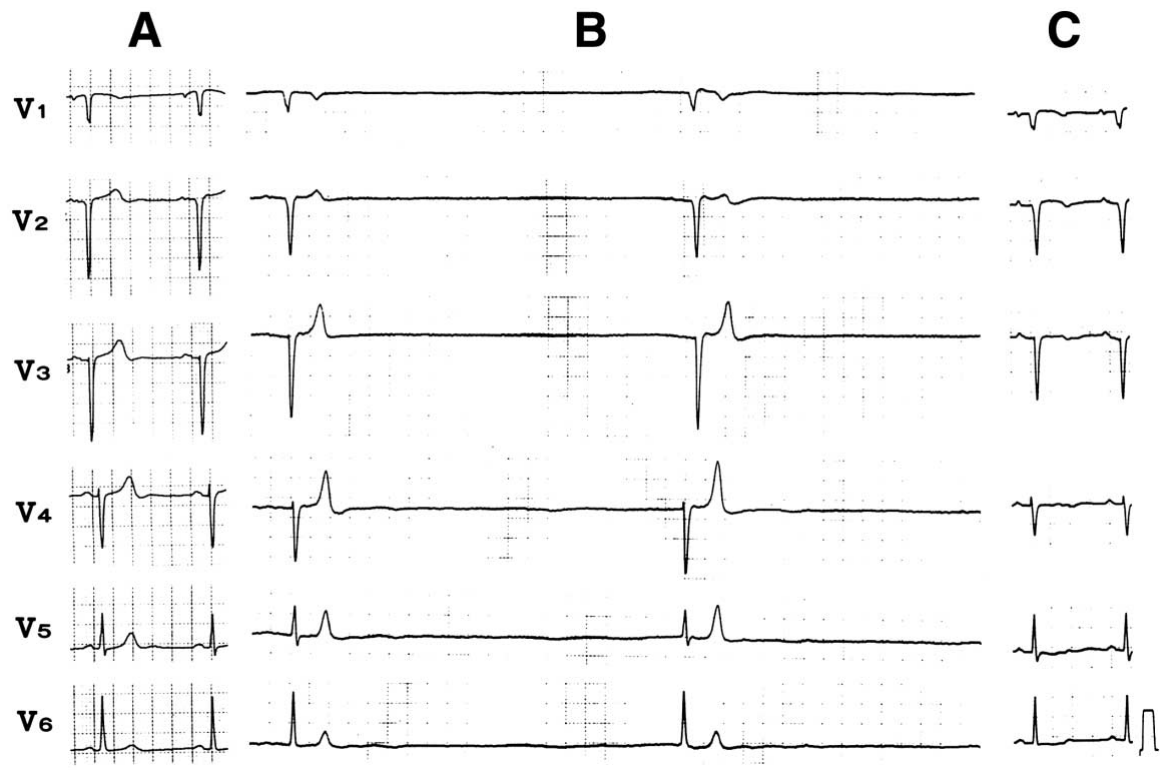

Fig 1. (A) Electrocardiogram (ECG) recorded 16 days before admission, showing poor R-wave progression in the precordial leads due to previous anterior infarction. (B) ECG on arrival (middle), showing marked bradycardia and tented $\mathrm{T}$ waves. (C) ECG several hours after arrival, showing normal sinus rhythm with reduced height of $\mathrm{T}$ waves.

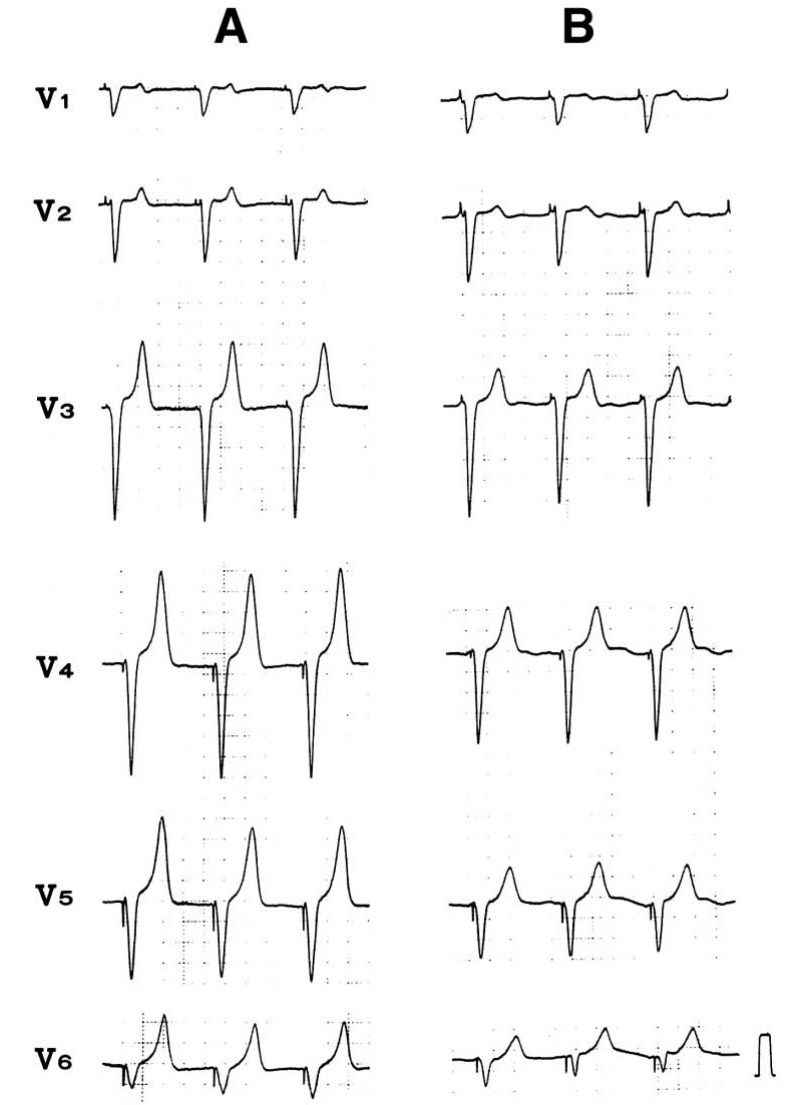

Fig 2. (A) Electrocardiogram (ECG) recorded immediately after the start of right ventricular pacing, showing tall and tent-shaped $\mathrm{T}$ waves. (B) ECG during right ventricular pacing recorded after normalization of the serum potassium level, showing a reduced amplitude of $\mathrm{T}$ waves.

wave, or abnormalities of impulse formation and conduction such as sinoatrial (SA) block and widening of the QRS complex! In the present case, the patient's advanced age and ischemic heart disease may have precipitated the occurrence of marked bradycardia in spite of the relatively modest hyperkalemia. The disappearance of the $\mathrm{P}$ wave was probably due to SA block and/or sinoventricular conduction; that is, transmission of the sinus impulse from the SA node to the atrioventricular node without activation of the surrounding atrial myocardium? In this case, furthermore, hyperkalemia-induced peaked $\mathrm{T}$ waves were observed during right ventricular pacing as well as during spontaneous rhythm.

It is well known that both ACEI and NSAID may cause hyperkalemia and, in particular, NSAID-induced hyperkalemia is more likely to occur in aged and diabetic patients taking ACEI? ACEI and NSAID are commonly prescribed as antihypertensives ${ }^{4}$ and analgesic-antipyretics, 5 respectively, for patients with no apparent renal dysfunction, including the elderly. In the present case, cessation of both the drugs may have been unnecessary for maintenance of normal serum potassium. Cessation of NSAID alone may have been enough, but ceasing both drugs may more reliably prevent the accidental induction of hyperkalemia.

This case warns physicians that the combination of NSAID and ACEI can produce serious side effects in aged patients who frequently suffer from hypertension, diabetes mellitus, ischemic heart disease, and degenerative joint disease.

\section{References}

1. Fisch C: Electrocardiography. In: Braunwald E, editor. Heart disease: a textbook of cardiovascular medicine, 5th edn. Philadelphia: WB Saunders, 1997: 141-142

2. Hayashi H, Hirai M: Atrial excitation and its disappearance. In: Harumi K, Arita M, Sugimoto T, Toyama J, Hashimoto K, editors. Rinsyo Shindengaku (Modern electrocardiology). Tokyo: Maruzen, 1993: 329-346 (in Japanese)

3. Whelton A, Hamilton CW: Nonsteroidal anti-inflammatory drugs: effects on kidney function. J Clin Pharmacol 1991; 31: 588-598

4. Hawkins DW, Hall WD, Douglas MB, Cotsonis G: A multi-center analysis of the use of enalapril and lisinopril in elderly hypertensive patients. J Am Geriatr Soc 1994; 42: 1273-1276

5. Hanlon JT, Fillenbaum GG, Studenski SA, Ziqubu-Page T, Wall WE Jr: Factors associated with suboptimal analgesic use in communitydwelling elderly. Ann Pharmacother 1996; 30: 739-744 